\title{
Low Grade Appendiceal Mucinous Neoplasms- A Short Case Series with Review of Literature
}

\author{
S. Salapathi*, B. Rajeshwari, Sadiya Niamath and Mitra Ghosh \\ Department of Histopathology, Apollo Specialty Hospitals, Vanagaram, Chennai -600095, India
}

\begin{abstract}
Low-grade appendiceal mucinous neoplasm (LAMN) is a rare malignancy and is found in less than $0.3 \%$ of appendectomy specimens. Patients with LAMN can present with abdominal pain and mimic clinically as appendicitis, intussusception or obstruction, hence high clinical suspicion is needed for correct diagnosis and management. The exact nature and nomenclature of these tumors when they disseminate into the peritoneum is controversial. The prognosis of LAMN is highly dependent on the presence or absence of neoplastic epithelium outside the appendix. Tumors with extra appendiceal acellular mucin in the right lower quadrant only rarely recur but tumors with extra appendiceal mucin and neoplastic epithelium recur more often. LAMNs with diffuse peritoneal seeding of neoplastic epithelium and mucin have a progressive clinical course that frequently results in death of the patient. Hence whenever a case of mucinous neoplasm of the appendix is encountered, it is essential that the entire appendix be submitted for histopathologic examination and should be thoroughly evaluated for presence of extra appendiceal mucin and neoplastic epithelium, and also for the type of invasion in order to obtain the correct diagnosis and to predict the risk of recurrence. We present here three cases of low grade appendiceal tumors with their varied clinical presentation and histopathological findings, for their rarity and clinical importance.
\end{abstract}

\section{Keywords: Mucinous Neoplasms, Appendix, Pseudomyxoma Peritonei}

\section{Introduction}

Appendiceal mucinous tumors include a spectrum of tumors ranging from microscopically benign to overtly malignant tumors. One of these include low grade appendiceal mucinous tumors (LAMN) which are the subject of considerable debate. Though these tumors have rather innocuous gross and microscopic appearance, they can penetrate into or through the appendix wall, rupture the appendix, and ultimately disseminate to the peritoneal cavity as pseudomyxoma peritonei (PMP). ${ }^{[1]}$ These neoplasms are more commonly diagnosed in men, particularly in the sixth decade of life. Patients with LAMN can present with abdominal pain and mimic clinically as appendicitis, intussusception or obstruction. Approximately $15-20 \%$ of LAMNs are incidental findings in patients undergoing surgery for unrelated conditions. $[1,2,3]$ Complications of LAMN include intussusception, obstruction, volvulus, small bowel obstruction, rupture, and PMP. ${ }^{[4,5,6]}$

\section{Case Report}

Case 1: A 53-year-old gentleman presented with complaints of abdominal pain for two days. There was no history of vomiting or loose stools. Clinical diagnosis of acute appendicitis was made. On examination patient was afebrile and tachycardic. Abdomen examination revealed tenderness in right iliac fossa with guarding. Baseline laboratory investigations done total and differential leucocyte counts were within normal limits.

Ultrasound (USG) abdomen was not done. Contrast enhanced computed tomography (CECT) done showed fluid filled bulky appendix with maximum diameter measuring $20 \mathrm{~mm}$ with peri-appendiceal fat stranding and narrowing at the base of appendix (Figure 1A). Minimal free fluid was seen in right iliac fossa. He underwent laparoscopic adhesiolysis with appendicectomy and the specimen was sent for histopathological examination. Grossly appendix with mesoappendix measured $7 \mathrm{~cm}$ in length. External surface showed congestion and tip of appendix was dilated with luminal diameter measuring $2 \mathrm{~cm}$. Cut surface of the appendix showed thickened wall near the tip, with a cystically dilated lumen filled with mucin. Rest of appendix and resected end of the appendix were grossly unremarkable.

On microscopy, sections showed cystically dilated tip of appendix lined by single layer of columnar epithelium with nuclear stratification, with elongated nuclei, showing prominent nucleoli and scant cytoplasm. Scattered apical mitosis noted. There were areas of neutrophilic infiltration over the ulcerated area, and partial disruption of muscularis. Focal pools of extracellular mucin were seen 
in the subserosa with no neoplastic epithelium (Figure 1B to 1D). There was no evidence of dysplastic epithelium or invasive malignancy elsewhere in the whole of appendix including resected end. Patient is on regular follow up for two years now with no evidence of recurrence or any evidence of Pseudomyxoma peritonei.

Case 2: A31-year-old gentleman presented to the emergency department with complaints of severe abdominal pain since 1day with history of one episode of vomiting and loose stools. There was history of fever for 3 days. Patient had recurrent episodes of similar abdominal pain for last 6 months and had multiple evaluations elsewhere since then and had exploration for perforated appendicitis. Baseline laboratory investigations including total and differential leucocyte counts were within normal limits.

USG abdomen was not done. CECT abdomen showed fluid filled bulky appendix noted with maximum diameter measuring $16 \mathrm{~mm}$. Peri-appendiceal fat stranding and fascial thickening was seen with narrowing of the base of appendix (Figure 2A). Intra-operatively there were omental adhesions, and appendix was adherent to the terminal ileum and caecum in retrocecal position. Appendicectomy was done. Grossly, appendix measured $7.2 \mathrm{~cm}$ in length. External surface showed congestion and adherent fat. Cut surface showed dilated lumen near the tip measuring $1.4 \mathrm{~cm}$ in diameter and lumen filled with mucoid material. No solid mass lesion was identified grossly. Proximal to the tip, there was a defect in the muscular layer suggestive of perforation. Resected end of the appendix was grossly unremarkable.

Microscopy showed appendix with mildly dilated lumen near the tip, lined by hyperplastic epithelium with low grade dysplasia, characterized by lining mucosa with basally located pencil shaped columnar nuclei, stratification of nuclei, mild nuclear atypia and scattered apical mitosis (Figure 2B to 2D). The dysplastic focus measured $0.5 \mathrm{~cm}$ in greatest dimension and was situated just below the tip. There was no evidence of invasion into the muscularis layer. Lumen contained scant mucin. Mucin stain by combined Periodic acid-Schiff (PAS) and Alcian blue showed loss of goblet cells in the dysplastic epithelium (Figure 3A\&3C), and highlighted the stromal mucin (Figure 3B). Proximal to the dilated lumen there was a perforated area with muscular wall defect, filled with dense mixed inflammatory infiltrate extending into subserosa and into mesoappendiceal fat. Foci of tiny mucinous pools containing neutrophils, without neoplastic epithelium seen in the subserosa near the perforated area. There was no evidence of dysplastic epithelium or invasive malignancy elsewhere in the whole of appendix including resected end. Serosa showed inflammatory exudate near perforated area with tiny mucinous pools situated just below the surface. Patient is on regular follow up for one year with no evidence of recurrence or any evidence of Pseudomyxoma peritonei.

Case 3: 61-year-old male, a known diabetic, presented to an outside hospital with abdominal pain on and off for 20 days and mild abdominal distension. There was history of altered bowel habits for last 7 days. There was no history of vomiting or fever. On examination abdomen was distended with mild tenderness in the right iliac fossa.

Ultrasound abdomen showed a mass lesion in the ileocaecal area, and appendix could not be visualized separately. Omental thickening was also noted. Initially omental biopsy was done and sent for histopathological examination which grossly showed mucoid areas. Microscopy showed predominantly fragments of thick mucin with few atypical glands floating within the mucin as well as lining the mucin fragments (Figure 4A). The glands showed nuclear stratification with mildly pleomorphic vesicular nuclei and tiny nucleoli. Rare mitosis was seen. The case was reported as Pseudomyxoma peritonei, and suggested to rule out appendiceal mucinous neoplasm. Subsequently patient underwent right hemicolectomy and specimen was sent for histopathological examination. Grossly the specimen measured $46 \mathrm{~cm}$ in length consisting of terminal ileum, caecum and proximal colon. External surface of caecum showed an exophytic mucinous lesion with dense adhesions, appeared to be arising from the appendix. Cut surface of the lesion showed mucinous areas with no solid areas. Appendix appeared to be distorted and only a tiny portion of appendix seen measuring $1 \times 0.5 \mathrm{~cm}$. Tip of the appendix could not be visualized separately from the adherent mucinous mass. The serosal aspect of ileocecal area and surrounding pericolic fat showed adherent thick mucin. Mucosa of ileum, caecum and proximal colon were unremarkable with no intraluminal growth or ulceration. Microscopy from the exophytic periappendiceal mass showed fibrous septae with pools of extracellular mucin and strips of neoplastic epithelium showing columnar cells with mildly pleomorphic elongated nuclei with stratification, loss of polarity and scattered apical mitosis (Figure 4B \&4C). The neoplastic epithelium showed focal pushing invasion into the fibrous stroma with no evidence of desmoplasia. A portion of unremarkable appendix with no dysplastic epithelial lining was seen adherent to the mass. Serosa of appendix, caecum and colon including subserosa of appendix showed pools of extracellular mucin with tiny strips of low-grade dysplastic epithelium. The mucosa of entire colon and ileum appeared unremarkable with no evidence of dysplasia. Fragments of omentum 
and peritoneal tissue sent separately also showed pools of extracellular mucin with strips of low-grade dysplastic epithelium. Regional lymph nodes showed reactive changes without tumor deposits. A final diagnosis of "Low grade mucinous neoplasm with Pseudomyxoma peritonei, and possibility of appendicular origin" was made. The patient was lost for further follow up. After two years, ascitic fluid sample was received from an outside centre for cytological examination which showed strips of lowgrade dysplastic epithelium with thick mucin fragments, suggestive of persistent PMP which was subsequently managed with peritonectomy (Figure 4D).

\section{Discussion}

Low-grade appendiceal mucinous neoplasm (LAMN) is a rare malignancy accounting for $1 \%$ of gastrointestinal neoplasms and is found in less than $0.3 \%$ of appendectomy specimens. ${ }^{[4,5,6]}$ According to WHO classification of appendiceal tumors, the term low-grade appendiceal mucinous neoplasm (LAMN) is used for low-grade mucinous tumors that reveal pushing invasion, and mucinous adenocarcinoma is used for high-grade appendiceal tumors and ones with conventional features of tissue invasion like irregular gland proliferation associated with stromal desmoplasia. ${ }^{[7,8]}$

Historically there has been considerable controversy in terminology of mucinous tumors of appendix. Earlier, low-grade appendiceal mucinous tumors were classified as "malignant mucoceles" or "grade I, noninvasive, papillary adenocarcinoma of the appendix". ${ }^{[9-13]}$ Later, these lesions were reclassified as benign neoplasms and the term cystadenoma was applied to denote their resemblance to colonic adenomas and to reflect their low-grade cytologic features, lack of destructive invasion, and benign clinical course. ${ }^{[2,14,15]}$

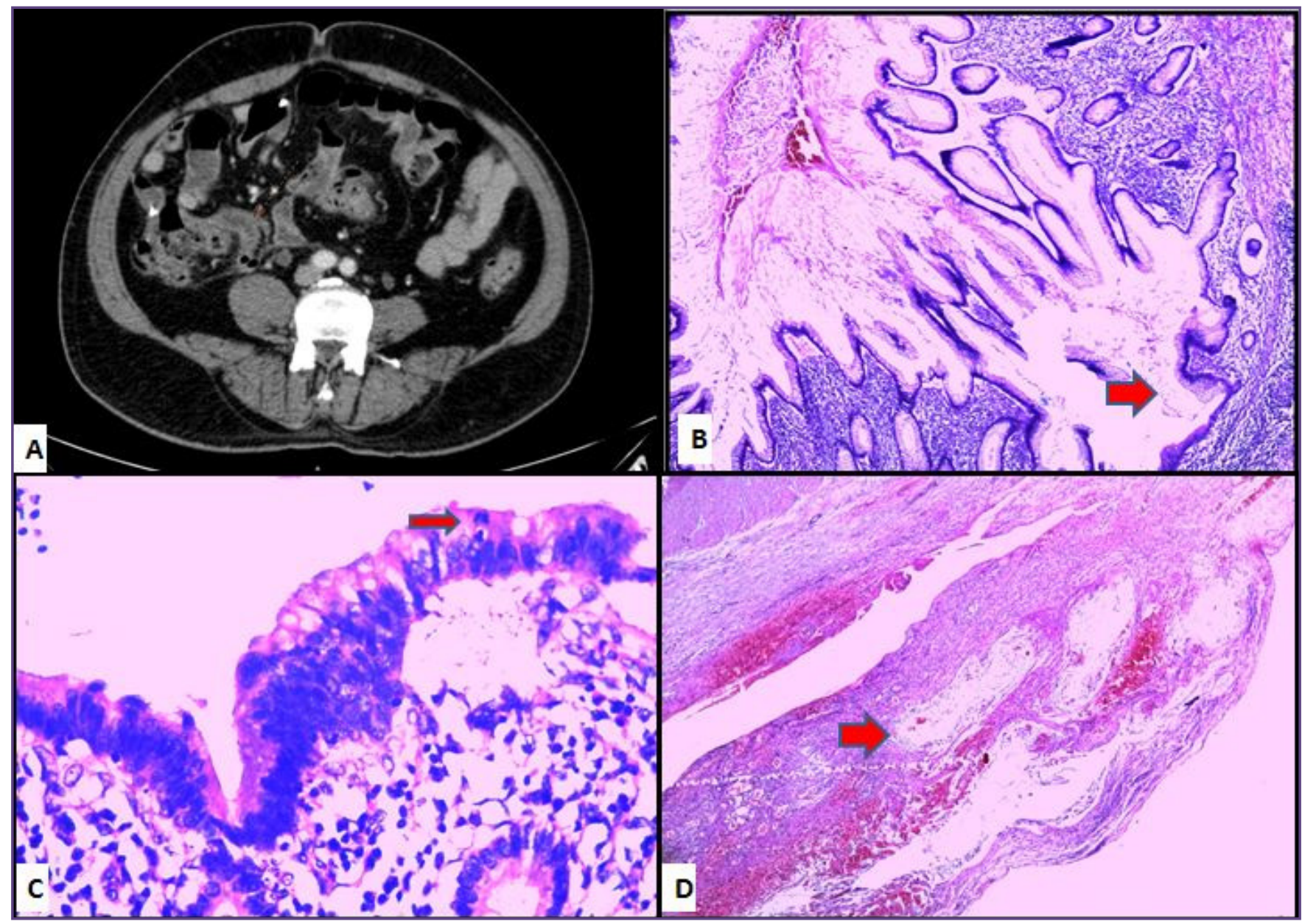

Fig. 1: A. CT scan abdomen of case 1 with contrast showing fluid filled bulky appendix (arrow) with surrounding periappendiceal fat stranding/facial thickening. B. Cystically dilated appendix lumen filled with mucin and wall lined by low grade dysplastic epithelium (arrow). Hematoxylin(H) \& eosin(E) stain, 40x. C. Low grade dysplastic mucinous epithelium with scattered apical mitosis (Arrow), 400x. Extracellular mucin without neoplastic epithelium in the subserosa (Arrows), 100x. 


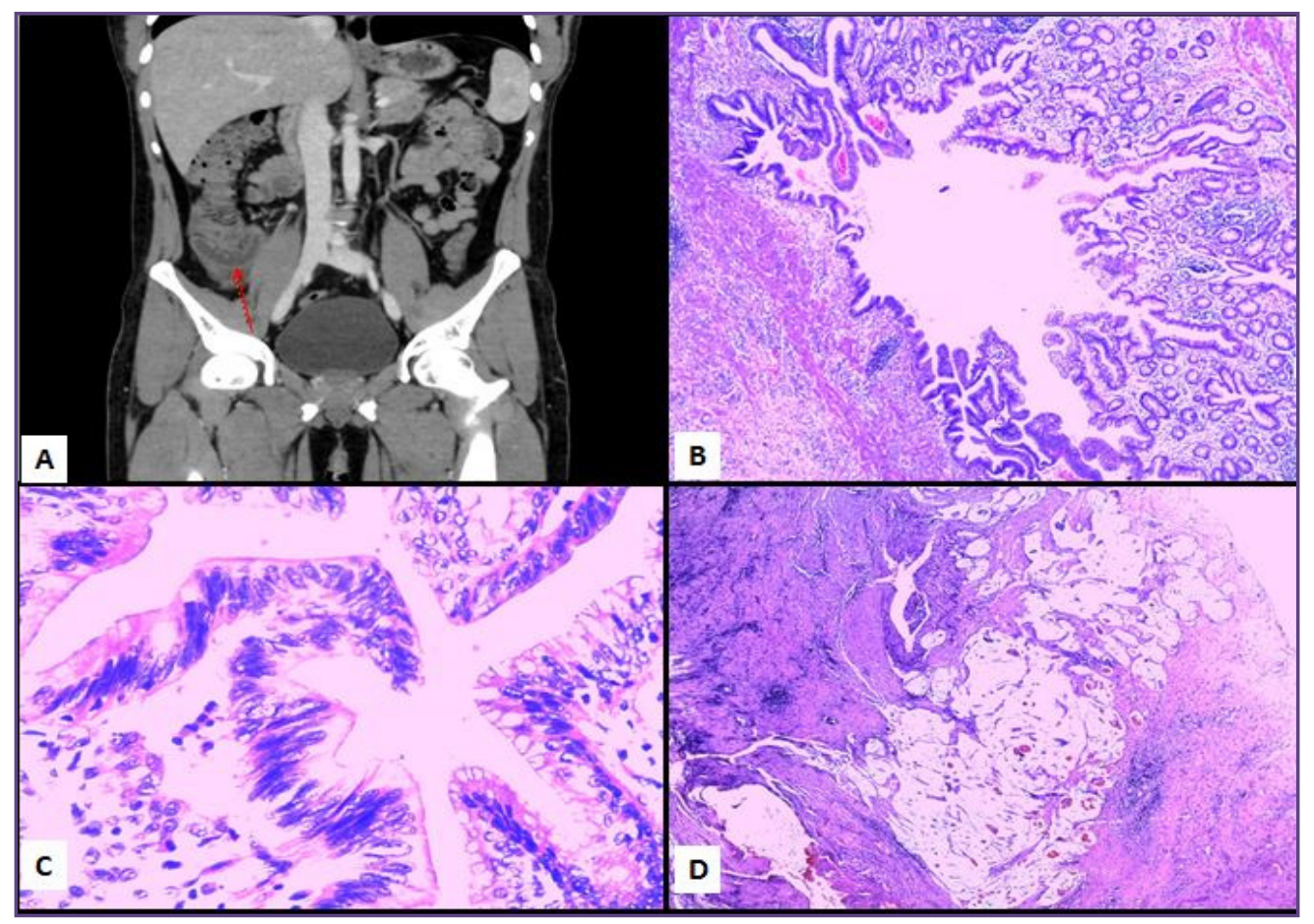

Fig. 2: A.CT scan abdomen with contrast of case 2 showing fluid filled bulky appendix with periappendiceal fat stranding (Arrow). B. Dilated appendix lumen lined by low grade dysplastic epithelium (Arrows). H \& E stain, 40x. C. Low grade dysplastic mucinous epithelium with nuclear crowding, elongated pencil shaped nuclei and mild nuclear atypia, 400x. D. Mucinous pools without neoplastic epithelium in the subserosa (Arrow), 100x.

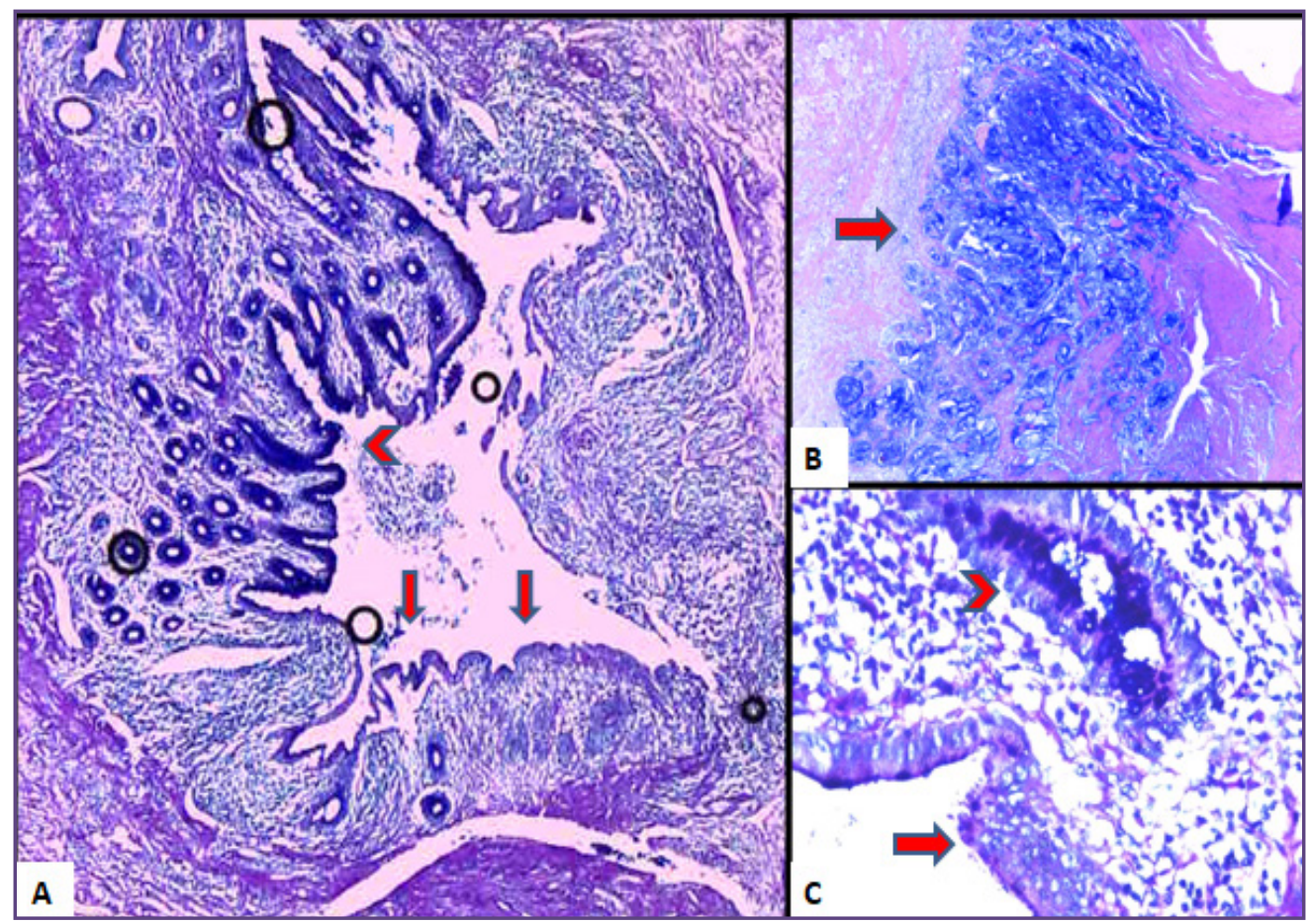

Fig. 3A \&C: Combined PAS-Alcian blue mucin stain shows loss of goblet cells in dysplastic epithelium (arrows) compared to non-neoplastic appendix mucosa (arrow head), and in 'B' highlighting blue stromal mucin pools (arrow). 


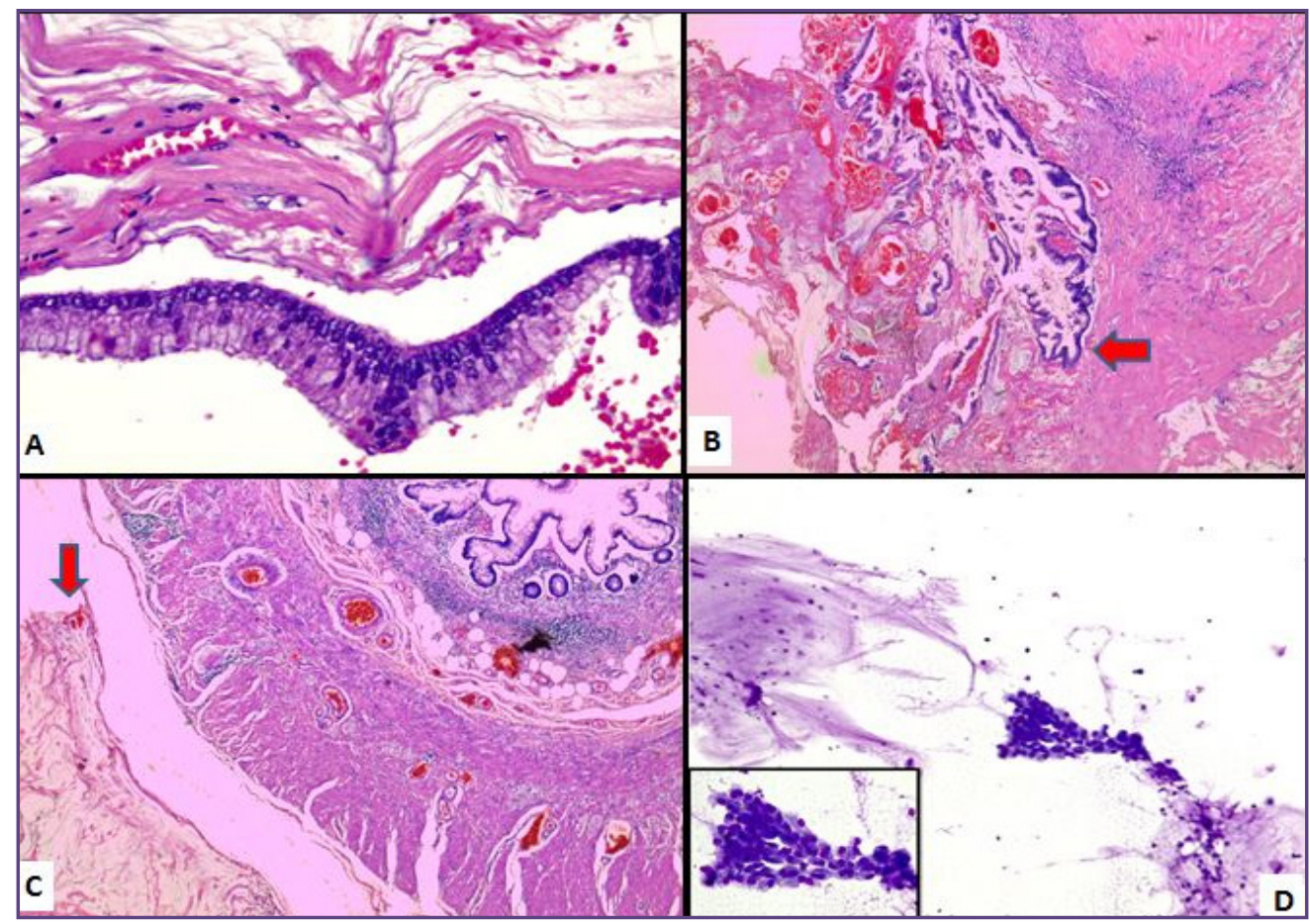

Fig. 4: A. Omental tissue with extracellular mucin and low grade dysplastic mucinous epithelium. H \& E stain, 100x. B. Periappendiceal mass showing pools of extracellular mucin and low grade dysplastic mucinous epithelium with pushing invasion into stroma (Arrow), 100x. C. Unremarkable portion of appendix with serosal extra appendiceal mucin (Arrow). 100x. D. Ascitic fluid cytology showing low grade dysplastic epithelium and mucin fragments. MGG stain, 100x. Inset shows high power view of the dysplastic epithelium.

Some authors proposed the term "mucinous tumor of uncertain malignant potential" to the tumors that appear to push deeply into the appendix wall but do not show dissemination beyond the appendix. ${ }^{[7,16]}$ Misdraji, in 2003, proposed the term "low-grade appendiceal mucinous neoplasm (LAMN)" to describe confined appendiceal neoplasms with or without pushing invasion, which explains their indolent but progressive malignant behavior yet absence of infiltrative invasion or high-grade cytology. ${ }^{[2,17]}$

The tumors associated with extra appendiceal mucin were classified by Pai and colleagues, as "low-grade mucinous neoplasm with low risk of recurrence" if the mucin located outside the appendix was acellular or "low-grade mucinous neoplasm with high risk of recurrence" if it contained neoplastic epithelium. ${ }^{[3]}$ In the 2010 WHO classification of appendiceal tumors, the term LAMN was incorporated into the spectrum of appendiceal adenocarcinomas describing them as low grade mucinous adenocarcinoma with pushing rather than infiltrative invasion. ${ }^{[1,8]}$

Activating mutations in Guanine Nucleotide binding protein, Alpha Stimulating activity polypeptide (GNAS) gene are seen in $50 \%$ of LAMNs and in the cell lines, this was associated with increased expression of the mucins MUC2 (Mucin 2) and MUC5AC (Mucin 5AC). GNAS mutations play an important role in the prominent mucin production that is a hallmark of LAMNs and PP. ${ }^{[7]}$

On USG imaging mucinous neoplasms appear as an encapsulated, elongated or ovoid cystic lesion in the appendix with an internal onion-skin appearance, which represents lamellated mucin and is considered pathognomonic. ${ }^{[17]}$ On CT, an appendix with a diameter of more than $15 \mathrm{~mm}$, soft tissue mass, wall thickening or irregularity should raise the suspicion of mucinous neoplasm. ${ }^{[18]}$ In Magnetic resonance imaging (MRI), mucinous neoplasms appear as hyperintense tubular distention of the appendix on T2-weighted images. Contrast-enhanced MRI may show smooth mucosal enhancement in simple lesions, whereas nodules and solid components are seen in adenomas, LAMNs and adenocarcinomas. Peri-appendiceal enhancement may be seen when there are adhesions or extraluminal mucin. The radiological differential diagnosis includes acute appendicitis when there is inflamed and perforated mucocele. Simple mucocele may appear radiologically similar to cystic lymphangioma, mesenteric cyst, enteric duplication cyst or retroperitoneal tumor. ${ }^{[19]}$ 
Histologically, LAMN is characterized by a villous or flat proliferative intestinal-type mucinous epithelium with low-grade cytologic features. ${ }^{[2,20,21]}$ These tumors may be confined to the mucosa, in which case they are classified as "adenomas," but they may also penetrate deeply into, or through, the appendiceal wall and disseminate to the peritoneal cavity, resulting in a clinical syndrome known as pseudomyxoma peritonei. ${ }^{[7]}$ Although LAMNs confined to the appendiceal lumen do not show definitive malignant features, they can proliferate outside the appendix in a malignant fashion and result in the development of pseudomyxoma peritonei, a life-threatening complication with 45\% 10-year survival. ${ }^{[22,23]}$ PMP was originally described by Werth in 1884, who attributed the abdominal mucin to an ovarian cyst. Fraenkel, in 1901 recognized PMP in the setting of an appendiceal tumor. ${ }^{[1,24]}$ LAMNs are also associated with diverticula, herniations, dissections, and rupture. ${ }^{[4,25]}$ Appendiceal mucinous neoplasms and peritoneal metastasis usually express cytokeratin 20, CDX2 and MUC2. In contrast, MUC1 is typically negative in adenomas, although it may be positive in adenocarcinomas. ${ }^{[7]}$

LAMNs that are confined to the appendix, without extraappendiceal mucin, are essentially cured by appendectomy similar to adenoma. ${ }^{[1]}$ Right hemicolectomy offers no additional benefit over appendectomy alone for patients with LAMN, even those with extra appendiceal mucin and neoplastic epithelium. Tumors that have disseminated widely throughout the peritoneal cavity are treated either by debulking or, more aggressively, with multiple peritonectomies and heated intraperitoneal chemotherapy. ${ }^{[7,27,28]}$

The prognosis of LAMN is highly dependent on the presence or absence of neoplastic epithelium outside the appendix. ${ }^{[2,3,7,16]}$ The clinical course is progressive when it is widely disseminated in the peritoneum. However, when the tumor is limited to the appendix or, when ruptured, confined to the right lower quadrant, the prognosis is related to the presence or absence of extra appendiceal mucin and in particular of neoplastic mucinous epithelium outside the appendix ${ }^{[1,2,3,16]}$ LAMNs associated with acellular mucin confined to the right lower quadrant carry a very low risk of recurrence or progression to PMP, whereas those associated with mucin and neoplastic epithelium in the right lower quadrant carry a relatively high risk of recurring as disseminated disease. ${ }^{[1]}$ Since the possibility of peritoneal recurrence in patients with ruptured LAMNs with acellular mucin on the periappendiceal serosa is high, these patients should be followed closely to ensure that localized or diffuse PMP does not develop. ${ }^{[7]}$ Radiographic imaging of the abdomen and pelvis is often the preferred method of surveillance. ${ }^{[7]}$

Yantiss et al and Pai et al studied 65 and 116 cases of appendiceal mucinous tumours respectively, and concluded that appendiceal mucinous tumors with extra appendiceal acellular mucin in the right lower quadrant only rarely recur but that tumors with extra appendiceal mucin with neoplastic epithelium recur more often. In contrast, LAMNs with diffuse (nonlocalized) peritoneal seeding of neoplastic epithelium and mucin have a rather progressive clinical course that frequently results in death of the patient unless managed aggressively. ${ }^{[3,7,26]}$ In our case series, two of our cases had presence of extra appendiceal acellular mucin limited to periappendiceal area who were managed with appendicectomy alone, didn't have any recurrence or PMP in two years of follow up. One patient with extensive extra appendiceal mucin with neoplastic epithelium around the right colon and omentum had PMP and recurred within two years of right hemicolectomy, and managed with subsequent peritonectomy and is on follow up. We are of the opinion and agree with Pai and colleagues that tumours associated with extra appendiceal mucin without neoplastic neoplasm limited to right lower quadrant should be labelled as "low grade appendiceal mucinous neoplasm with low risk of recurrence", and "low-grade mucinous neoplasm with high risk of recurrence" for those with mucin and neoplastic epithelium, as they clearly denote the prognosis and will help in further management.

\section{Conclusion}

LAMN is a rare appendiceal mucinous tumor with prognosis being dependent on the presence or absence of extra appendiceal mucin and neoplastic epithelium. Hence the evaluation of the appendix specimen for extra appendiceal mucin and neoplastic epithelium should be performed carefully to determine the prognosis and treatment of these tumors.

\section{Acknowledgements}

We sincerely thank Dr.K.Ramkumar, Senior consultant, Surgical gastroenterology department and Dr.Vishwanath M Pai, Senior cosultant, General surgery department for their clinical inputs and Dr.Sanjeeb Agarwal, Senior consultant, radiology department for providing radiology images. We sincerely acknowledge our technical staff, Mrs P Shivashankari, Mr R Bhuvaneshwaran, Mrs Lavanya Latha, Mrs J Rukmani, for their technical help.

\section{Funding}

Nil

\section{Competing Interests}

Nil 


\section{Reference}

1. Misdraji J. Mucinous epithelial neoplasms of the appendix and pseudomyxoma peritonei. Mod Pathol. 2015 ;28:67-79.

2. Misdraji J, Yantiss RK, Graeme-Cook FM, Balis UJ, Young RH. Appendiceal mucinous neoplasms: a clinicopathologic analysis of 107 cases. Am J Surg Pathol 2003; 27:1089-103.

3. Pai RK, Beck AH, Norton JA, Longacre TA. Appendiceal mucinous neoplasms: clinicopathologic study of 116 cases with analysis of factors predicting recurrence. Am J Surg Pathol 2009;33:1425-39.

4. Gonzalez HH, Herard K, Mijares MC. A Rare Case of Lowgrade Appendiceal Mucinous Neoplasm: A Case Report Cureus 2019;11: e3980. DOI 10.7759/cureus.3980

5. Ramaswamy V. Pathology of mucinous appendiceal tumors and pseudomyxoma peritonei . Indian J Surg Oncol. 2016, 7:258-67.

6. Padmanaban V, Morano WF, Gleeson E, Aggarwal A, Mapow BL, Stein DE et al. Incidentally discovered lowgrade appendiceal mucinous neoplasm: a precursor to pseudomyxoma peritonei. Clin Case Rep. 2016; 4:1112- 6.

7. Misdraji J. Epithelial neoplasms of Appendix. In: Odze and Goldblum's Surgical Pathology of the GI Tract, Liver, Biliary Tract, and Pancreas. 3rd edition. Philadelphia: Elsevier Saunders; 2015. p 779-91.

8. Carr NJ, Sobin LH. Adenocarcinoma of the appendix, In: Bosman FT, Carneiro F, Hruban RH, et al. editors. World Health Organization Classification of Tumours of the Digestive System. IARC Press: Lyon, France; 2010, pp 122-5.

9. McGregor JK, McGregor DD. Adenocarcinoma of the appendix. Surgery 1960;48:925-35.

10. Steinberg M, Cohn I Jr. Primary adenocarcinoma of the appendix. Surgery 1967;61:644-60.

11. Uihlein A, McDonald JR. Primary carcinoma of the appendix resembling carcinoma of the colon. Surg Gynecol Obstet 1943;76: 711-4.

12. Wilson R. Primary carcinoma of the appendix. Am J Surg 1962; 104:238-49.

13. Woodruff R, McDonald JR. Benign and malignant cystic tumors of the appendix. Surg Gynecol Obstet 1940;71:750-5.

14. Gibbs NM. Mucinous cystadenoma and cystadenocarcinoma of the vermiform appendix with particular reference to mucocele and pseudomyxoma peritonei. J Clin Pathol 1973;26:413-21.

15. Higa E, Rosai J, Pizzimbono CA, Wise L. Mucosal hyperplasia, mucinous cystadenoma, and mucinous cystadenocarcinoma of the appendix: a re-evaluation of appendiceal 'mucocele.' Cancer 1973; 32:1525-41
16. Carr NJ, McCarthy WF, Sobin LH. Epithelial noncarcinoid tumors and tumor-like lesions of the appendix: a clinicopathologic study of 184 patients with a multivariate analysis of prognostic factors. Cancer 1995; 75:757-768

17. Caspi B, Cassif E, Auslender R, Herman A, Hagay Z, Appelman Z. The onion skin sign. a specific sonographic marker of appendiceal mucocele. J Ultrasound Med 2004; 23: $117-21$

18. Persaud T, Swan N, Torreggiani WC. Giant mucinous cystadenoma of the appendix. Radiographics 2007; 27: 553-57.

19. Tirumani SH, Fraser-Hill M, Auer R, Shabana W, Walsh C, Lee $\mathrm{F}$ et al. Mucinous neoplasms of the appendix: a current comprehensive clinicopathologic and imaging review. Cancer Imaging 2013; 13: 14-25.

20. Li X, Zhou J, Dong M, Yang L. Management and prognosis of low-grade appendiceal mucinous neoplasms: A clinicopathologic analysis of 50 cases. Eur J Surg Oncol. 2018;44:1640-5.

21. McDonald JR, O'Dwyer ST, Rout S, Chakrabarty B, Sikand $\mathrm{K}$, Fulford PE et al. Classification of and cytoreductive surgery for low-grade appendiceal mucinous neoplasms. $\mathrm{Br}$ J Surg 2012;99:987-92.

22. Yu XR, Mao J, Tang W, Meng XY, Tian Y,Du ZL. Lowgrade appendiceal mucinous neoplasms confined to the appendix: clinical manifestations and CT findings. J Investig Med 2020;68:75-81.

23. Nishikawa G, Sekine S, Ogawa R, Matsubara A, Mori $\mathrm{T}$, Taniguchi $\mathrm{H}$ et al. Frequent GNAS mutations in lowgrade appendiceal mucinous neoplasms. $\mathrm{Br} \mathrm{J}$ Cancer 2013;108:951-8.

24. Werth R. Klinische und anatomische untersuchungen zur lehre von den bauchgeschwuelsten und der lapartomie. Arch f Gynaek 1884;24:100-18.

25. Misdraji J, Young RH: Primary epithelial neoplasms and other epithelial lesions of the appendix (excluding carcinoid tumors). Semin Diagn Pathol. 2004, 21:120-33.

26. Yantiss RK, Shia J, Klimstra DS, Hahn HP, Odze RD, Misdraji J. Prognostic significance of localized extraappendiceal mucin deposition in appendiceal mucinous neoplasms. Am J Surg Pathol. 2009;33:248-55.

27. Gonzalez-Moreno S, Sugarbaker PH. Right hemicolectomy does not confer a survival advantage in patients with mucinous carcinoma of the appendix and peritoneal seeding. Br J Surg 2004; 91:304-11.

28. Foster JM, Gupta PK, Carreau JH, Grotz TE, Blas JV, Gatalica $\mathrm{Z}$ et al. Right hemicolectomy is not routinely indicated in pseudomyxoma peritonei. Am Surg 2012;78:171-7.

\section{*Corresponding author:}

Dr. S. Salapathi, Associate Consultant, Department of Histopathology, Apollo Specialty Hospitals,

Vanagaram. Kil Ayanambakkam, Chennai - 600095. India.

Email: salapsdr@gmail.com

Date of Submission : $17 / 10 / 2020$

Financial or other Competing Interests: None. 